\title{
Slings operations work very differently from mesh sheet implantations and should not be banned
}

\author{
(D) PETER PETROS
}

University of Western Australia, School of Mechanical and Mathematical Engineering

\section{ABSTRACT}

Baroness Cumberlage has overreacted banning midurethral slings along with mesh sheets placed behind the vagina.

We should all support the banning of mesh sheets. Midurethral and other slings work differently from mesh which does not correct the prolapse. Mesh sheets just block the prolapse from descending. In the process the vagina is fibrosed. This can trap nerves to cause pain, and interfere with the closure and opening mechanisms of the bladder to cause massive incontinence. These mechanisms rely on a vagina which has adequate elasticity.

The midurethral sling operation is the most validated operation in surgical history with more than 1,000 publications attesting to its safety and efficacy. It was tested with animals and seven prototype operations prior to release in 1996, 10 years after the animal experiments began.

Slings work differently. They have very little contact with the vagina and so don't fibrose it. Nerve trapping is rare. Banning slings leaves women with very few options.

Hopefully the authorities will re-examine mesh slings and restore them. Of significant help to this are 3-year data from sling operations using next generation tapes with no erosions at all in that 3 years.

Keywords: Midurethral slings; ban; mesh sheets

The persistent media campaign against mesh products in the United Kingdom (UK) successfully resulted in the banning earlier this year of all mesh slings and mesh kits by the UK government. Unlike the Food and Drug Administration (FDA) where suppliers of mesh kits were put on notice to provide the data to justify their continued sale, the UK government instituted the Cumberlage report, ${ }^{1}$ and acted on its recommendations. As the co-inventor of the now discontinued midurethral sling (MUS), the subject of this editorial, I fully endorse the recommendations for thorough premarket testing on any implantable device, for that is exactly the course which the late Ulf Ulmsten and I followed with the
MUS. I also endorse the ban against mesh sheets which did not follow the exhaustive testing of the MUS.

The Cumberlage report, ${ }^{1}$ unfortunately, is not based or robust scientific data. It consisted of opinions mainly from non-experts, including a journalist. It does not take into consideration the vast 10-year clinical and scientific background of the MUS. Apparently not considered were well reasoned scientific opinions from expert committees from almost every learned body, which generally concluded that the benefits of slings far outweighed their complications and, even years ago, that mesh sheet surgery required more proof. 
The Cumberlage report made no differentiation between mesh sheets and slings. Mesh sheets applied behind the vagina to block descent of organs is a very different methodology from slings.

Mesh sheets fibrose vagina, may trap nerves, leading to pain, may inhibit movements essential for bladder closure and evacuation. ${ }^{2}$ Nor do mesh sheets actually cure the prolapse. Simple 2D ultrasound shows that the prolapse is still there behind the mesh. The mesh blocks it coming down. In contrast, slings, using narrow tapes less than $1 \mathrm{~cm}$ in width, attach organs directly to the skeleton and create new collagen to restore structure and function. ${ }^{3}$ Because they have very little contact with the vagina, slings avoid trapping nerves and preserve vaginal elasticity needed for bladder function. ${ }^{2}$ Complications typical of large mesh are rarely seen with MUS.

The Cumberlage report bundled MUS with mesh sheet surgery and banned both. It ignored that the MUS was the most validated operation in the history of surgery with a profound experimental and clinical base. In fact, the MUS was not released until 1996, 10 years after the first animal experiments started in 1986 at Royal Perth Hospital by the author, ${ }^{3}$ ironically, to test the safety and efficacy of the tape! ${ }^{2}$

The animal experiments ${ }^{3}$ were critical to the underlying hypothesis, that the cause of stress urinary incontinence (SUI) was ultimately due to collagen defects in the pubourethral ligaments (PUL); a tape placed in the exact position of PUL would harness the wound reaction from the tissues to create a collagenous neoligament. This "neoligament" principle was later extended to cure weakened ligaments such as cardinal, uterosacral, arcus tendineus fascia pelvis (ATFP) which caused prolapse, consistent with the three-level support system proposed by DeLancey's anatomical studies.

The animal experiments were analysed by the author and Professor John Papadimitriou, who is an acknowledged world expert on macrophages and inflammation research. Included in the animal testing was clinical monitoring over 3 months, radioactive Gallium studies, X-ray studies, regular biochemical and hematology testing of the animals, anatomical studies, biomechanical testing of the neoligament for breaking strength, extensive histology and bacteriology. ${ }^{3}$ The animal experiments proved that tape implantation was safe and would create a collagenous neoligament to reinforce weak ligaments.

The first prototype human operations underwent 5-year Royal Perth Hospital EC surveillance, between 1988 and 1993. Tapes were implanted in the position of the PUL. The prototype human studies were subjected to thorough clinical, bacteriological, radiological, hematological and biochemical observations.
Meanwhile Ulmsten, Nilsson and others performed their own parallel studies in Scandinavia. By the time the MUS was released in 1996, the MUS had undergone 10 years study for safety and efficacy under Ethics Committee surveillance in several international locations. Since 1996, the MUS has been the subject of $>1000$ scientific papers, (including 17-year data), $10,000,000$ MUS surgeries. It has been endorsed by almost every learned body. In contrast, mesh sheet surgery for prolapse has had very little premarket scientific testing.

The Cumberlage statement of inadequate premarket certainly applies to mesh sheets. It clearly cannot apply to slings, as implied in her statements banning them. ${ }^{1}$

The real reason for banning seemed to be anecdotal complications from many suffering women. This important issue was examined in detail in a previous International Urogynecology Journal (IUJ) editorial, Should surgeons continue to implant mesh sheets behind the vagina $?^{4}$ The editorial concluded that though mesh sheets worked well in many cases, reports of severe pain due to nerve entrapment and scar-induced disturbance of the control mechanisms of the bladder to cause massive urine loss "tethered vagina Syndrome," posed an important question: are severe disabling complications sufficient to prevent mesh sheets being done at all? Fortunately, it has been proven that mesh sheets are not necessary for repair of major prolapse. In a multicentre study of 616 women, Liedl et al. ${ }^{5}$ demonstrated that tensioned mini slings applied to cardinal and uterosacral ligaments in women with mostly $3^{\text {rd }}$ or $4^{\text {th }}$ degree uterine/apical prolapse were sufficient to achieve a $90 \%$ anatomical cure at 12 months. ${ }^{5}$ There was no vaginal excision. Dislocated or overstretched vagina was refashioned and re-attached.

The Cumberlage decision in the UK leaves women with poor outdated treatment options, "native tissue" vaginal repairs with up to $80 \%$ failure rates according to the prospect study or the Burch Colposuspension with all the complications which led to its demise a few short years after introduction of the MUS.

There are cogent scientific reasons why slings should not be discontinued. Ligaments are key elements in the three-level support system for organs. Collagen leaches out of the ligaments after the menopause to cause prolapse and incontinence. ${ }^{2} \mathrm{~A}$ tape placed along ligaments as in the MUS is the only validated surgical method which can create new collagen to repair organ support.

And finally, technical advances. Ninety percent cure rates are being achieved for ISD (Intrinsic sphincter defects) and SUI using TFS mini slings, with zero erosions at 3 years. ${ }^{6,7}$ 


\section{Ethics}

Peer-review: Externally peer-reviewed.

\section{DISCLOSURES}

Financial Disclosure: The authors declared that this study has received no financial support.

\section{REFERENCES}

1. Baroness Cumberlage Press conference speech, $8^{\text {th }}$ July 2020.

2. Petros PE, Ulmsten UI. An integral theory of female urinary incontinence. Experimental and clinical considerations. Acta Obstet Gynecol Scand Suppl 1990; 153: 7-31.

3. Petros P, Papadimitriou J. Evolution of midurethral and other mesh slings--a critical analysis. Neurourol Urodyn 2013; 32: 399-406.
4. Petros PEP, Liedl B, Gold D. Should surgeons continue to implant mesh sheets behind the vagina? Int Urogynecol J 2018; 29: 777-9.

5. Liedl $\mathrm{B}$, Inoue $\mathrm{H}$, Sekiguchi $\mathrm{Y}$, et al. Is overactive bladder in the female surgically curable by ligament repair? Cent European J Urol 2017; 70: 53-9.

6. Nakamura R, Yao M, Maeda Y, Fujisaki A, Sekiguchi Y. Retropubic tissue fixation system tensioned mini-sling carried out under local anesthesia cures stress urinary incontinence and intrinsic sphincter deficiency: 1-year data. Int J Urol 2017; 24: 532-7.

7. Nakamura R, Yao M, Maeda Y, Fujisaki A, Sekiguchi Y. Outpatient mid-urethral tissue fixation system sling for urodynamic stress urinary incontinence: 3-year surgical and quality of life results. Int Urogynecol J 2017; 28: 1733-8. 